\title{
La consulta de medicina tropical Tropical medicine consultation
}

\author{
L. Valerio ${ }^{1}, X$. de Balanzó ${ }^{2}$
}

\section{RESUMEN}

Las consultas de medicina tropical tienen sentido en entornos altamente tecnificados, en los que se dispone de pruebas complementarias específicas y de profesionales con experiencia en el trópico. Es decir, en hospitales de tercer nivel. Si estas consultas estuvieran situadas en hospitales de segundo nivel o en la atención primaria podrían considerarse como ineficientes o no justificables desde el punto de vista del volumen de pacientes atendidos. Sin embargo, existe un déficit asistencial por lo que respecta a actividades preventivas en viajeros o en inmigrantes recién llegados de países de renta baja con alta prevalencia de enfermedades importadas que son menos reconocidas en nuestro habitual medio sanitario.

Así, unidades de salud internacional que combinen actividades preventivas y curativas en una marco de provisión sanitaria pública y en una situación funcional entre el nivel hospitalario y el de la atención primaria, ofrecen un perfil más eficiente y adecuado para las características de la población española. Su implantación depende de la voluntad de los responsables político-sanitarios, de la organización de una cartera de servicios realista, de la existencia de monitorización del control de calidad y de la posibilidad de reinformación mediante trabajo en red informática.

Palabras clave. Salud internacional. Medicina tropical. Enfermedades importadas. Inmigración y atención primaria de salud. Unidad de salud internacional.

An. Sist. Sanit. Navar. 2006; 29 (Supl. 1): 97-104.

\begin{abstract}
Tropical medicine consultations are fully justified in settings with the latest modern technology, where specific complementary tests are available and there are professionals with experience in tropical questions. That is to say, in tertiary hospitals. If such consultations took place in secondary hospitals or in primary care, they could be considered inefficient or unjustifiable from the point of view of the volume of patients attended to. However, there is a care deficit with respect to preventive activities concerning travellers or immigrants who have recently arrived from countries with a low income and where there is a high prevalence of imported diseases that are less recognised in our normal health milieu.

Thus, international health units, which combine preventive and curative activities in a framework of public health provision and in a functional situation between the hospital level and that of primary care, offer a more efficient and suitable profile for the characteristics of the Spanish population. Their implementation depends on policy makers, the offer of a realistic portfolio of services, the existence of quality control monitoring and the possibility of managing information through a computer network.
\end{abstract}

Key words. International health. Tropical medicine. Imported diseases. Immigration and primary health care. International health unit.
1. Unidad de Salud Internacional del Barcelonès nord i Maresme. Servei d'Atenció Primària Sta. Coloma de Gramenet. Institut Català de la Salut. Barcelona.

2. Jefe del Servicio de Medicina Intensiva. Hospital de Mataró. Consorci Sanitari del Maresme. Mataró. Barcelona.

\section{Correspondencia:}

Lluís Valerio Sallent

C/ Jacint Verdaguer, 118

08923 Sta. Coloma de Gramenet

Barcelona

Tfno. 933922213

E-mail: lvalerio.bnm.ics@gencat.net 


\section{CONSIDERACIONES PREVIAS. ECONOMÍA Y ÉTICA}

Una verdadera consulta de medina tropical debe tener a su disposición desde camas de aislamiento hasta pruebas diagnósticas de alta complejidad técnica, pasando por el acceso a medicamentos extranjeros y vacunaciones amén de personal con conocimientos específicos en el ámbito de enfermedades infecciosas, salud comunitaria, enfermería, epidemiología y microbiología. Planteado en forma de pregunta, ésta sería: ¿cómo puede ser sostenible- eficiente -una unidad cuyos procesos asistenciales son de alta especificidad en un entorno de provisión sanitaria pública, es decir, de restricción presupuestaria?

Además de responder a la pregunta anterior, conviene aclarar que una consulta de medicina tropical debe ser éticamente aceptable en el sentido que no debe convertirse en un sistema sanitario hermético en miniatura -o arrogarse el papel de ONG- donde se atiende a inmigrantes eventualmente enfermos. El objetivo no es atender a casos exóticos sino atender a personas. Las actividades asistenciales deben insertarse con normalidad en la cartera de servicios de un hospital o de una consulta externa de proveedores del sistema nacional de salud.

El presente artículo propone unas orientaciones sobre la estructura de una "consulta de medicina tropical". Se basa en la experiencia acumulada en la organización y desarrollo de una Unidad de Salud Internacional que se relaciona con su entorno sanitario tanto a nivel de atención primaria como de atención hospitalaria en el ámbito sanitario del Barcelonès Nord $i$ Maresme (Barcelona), zona que aúna dos comarcas con una población total de unos 700.000 habitantes y donde existe una provisión en salud internacional basada en unidades interniveles -primero en Mataró y después en Sta. Coloma de Gramenetdesde 1984. De manera orientativa, en la figura 1 se muestra un resumen de la actividad asistencial durante el período 19992005 en la que la consulta prioritaria y creciente se centró en el consejo del viajero, atendiéndose un promedio de ochenta consultas anuales relacionadas con enfermedades importadas.

\section{PLANTEAMIENTO ORGANIZATIVO DE LA UNIDAD DE SALUD INTERNACIONAL}

\section{Elementos fundacionales o la estrategia}

\section{Relación de interniveles}

La inmigración y los desplazamientos internacionales turísticos y comerciales, con su crecimiento y demanda exponenciales, colapsan los servicios de sanidad exterior ministeriales o las unidades asociadas oficiales. Además, en muchas ocasiones dichos centros se encuentran aislados del sistema de salud de la comunidad autónoma e incurren en duplicidades, redundancias y, en definitiva, ineficiencia. Además, respondiendo a un modelo centralizado provincial, es frecuente que se encuentren a mucha distancia física de los núcleos de población con mayor número de consultas. Así, cada vez en mayor medida, los usuarios consultan con sus médicos de atención primaria por inexistencia de alternativas exentas de listas de espera y por la simplicidad de acceso (padrón). La mayor parte de las patologías subclínicas en inmigrantes son -y lo serán cada día más- detectadas en los exámenes de cribado de los médicos de familia: especialmente aumentarán los casos de infección tuberculosa latentes, enfermedades de transmisión sexual subclínicas, hepatopatías, helmintiasis, alteraciones de la serie roja (hemoglobinopatías) o de eosinofilias a estudio, por poner los problemas más frecuentes. Asimismo aumentará la actividad preventiva por lo que se refiere a consejos a viajeros, ya sean por retorno de inmigrantes a su país de origen, por desplazamientos vacacionales, laborales o de cooperación en viajeros autóctonos. Por lo tanto, la unidad de salud internacional debe plantearse con horizonte de ámbito sanitario, englobando los niveles primario y hospitalario. Las actividades preventivas así como el tratamiento de algunos procesos importados de menor complejidad se centrarán en la atención primaria y una parte de las infecciones clínicas importadas se tratarán en el hospital ${ }^{1}$. 


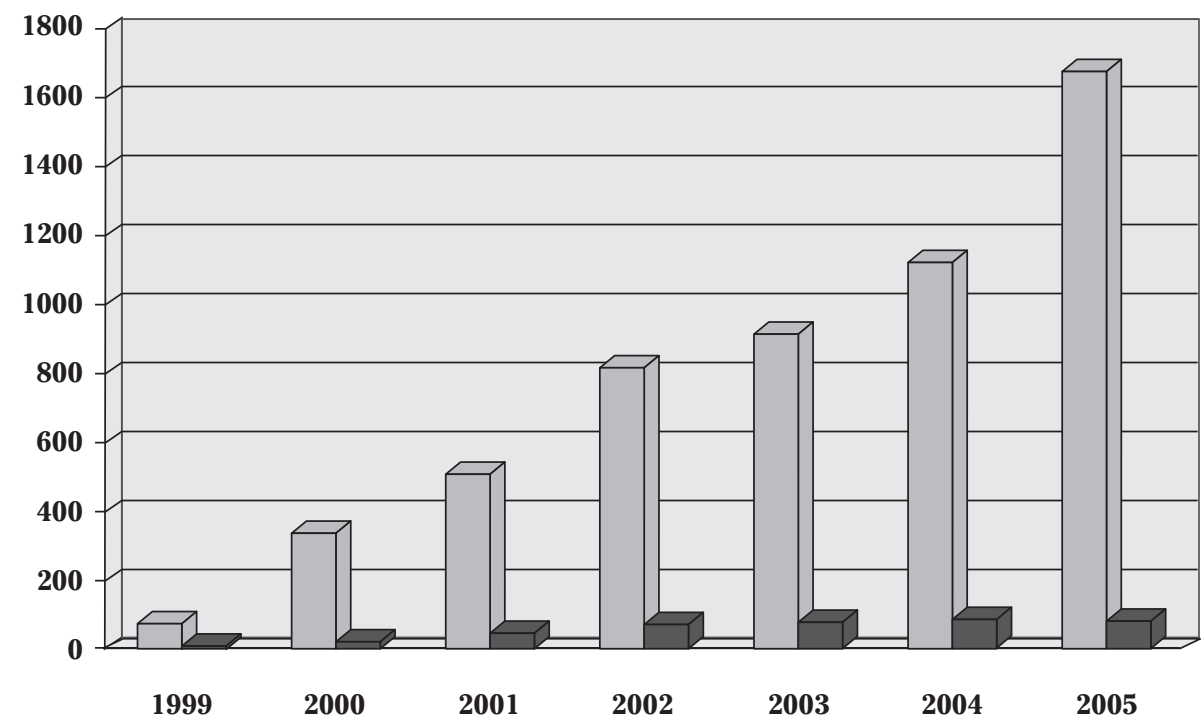

Figura 1. Actividad asistencial (1as visitas) de la USAI BniM 1999-2005.

En este sentido, los responsables asistenciales de la unidad deben ser percibidos más como consultores que como especialistas clásicos a los que se deriva mediante el correspondiente impreso. Los médicos de cabecera del ámbito definido deben conocerlos y debe existir una línea de teléfono o correo electrónico accesible a consultas. Los hospitales del ámbito, asimismo, deben tener un internista o infectólogo de referencia avezado en los problemas de salud internacional ${ }^{2}$ y un buen interlocutor en los servicios de diagnóstico por la imagen y de laboratorio (hematología y microbiología).

Además, deben existir relaciones con los servicios de sanidad de la comunidad autónoma correspondiente. El planteamiento fundacional de una unidad de salud internacional debería incluir la presentación y negociación con las autoridades sanitarias antes del inicio de sus actividades y rehuir presentarla como un hecho consumado.

La existencia de unidades interniveles reales depende de la disponibilidad de conexión a la red informática compartida entre los niveles implicados. Si no se dispone de ella de entrada, conseguirlo a medio plazo debe considerarse algo fundamental.

\section{¿Medicina tropical o actividades preventivas?}

El término medicina tropical tiene un origen colonial. Fue creado por los europeos que estudiaban las patologías presentes en un ecosistema que, por contraste con el europeo, parecía exótico. No obstante, desde los años 80 del pasado siglo, se desarrolló el concepto de salud internacional para abordar también la epidemiología, la salud pública y la medicina preventiva a un nivel global y de manera menos unidireccional y respetuosa para con los profesionales de los países de baja renta.

Así, en nuestra opinión, es mejor diseñar la consulta como unidad de salud internacional, donde se realicen actividades preventivas (consejo a viajeros, vacunación internacional), actividades curativas (atención a enfermedades importadas) 
y de promoción y protección de la salud (salud comunitaria). La complejidad y las necesidades inversoras iniciales son superiores pero, en definitiva, es más adecuado a las necesidades de los usuarios del siglo XXI y permite aumentar el volumen de pacientes atendidos -a expensas de las actividades preventivas en viajeros- hasta sobrepasar el volumen de actividad mínima a partir del que una unidad puede considerarse como factible -en Catalunya, el Departament de Salut situó el dintel mínimo de actividad en 1.000 primeras visitas al año. Las consultas de medicina tropical, en sentido estricto, sólo son sostenibles en lo alto de la pirámide asistencial, es decir en aquellos hospitales de nivel B con importantes núcleos de población inmigrante o viajera y, en todo caso, en los de nivel C (o tercer nivel según la terminología que se utilice $)^{3-5}$.

\section{Facturación a terceros}

En Catalunya, desde 2003, los usuarios de las actividades preventivas deben abonar una cantidad de dinero simbólica. No hay que tener esperanzas infundadas: la mayoría de las unidades están muy lejos de la autofinanciación y no debería crearse una unidad con criterios economicistas. Pero es importante decidir si la atención -considerada fuera de la cobertura de la seguridad social- debe ser totalmente gratuita o no. Desde luego, las actividades curativas no son facturables ${ }^{6}$. En la tabla 1 se exponen pros y contras de la facturación a terceros.

\section{¿Provisión pública o privada?}

Coexisten países donde las actividades preventivas en viajeros se realizan mayori- tariamente en el sector privado (Suiza, Francia, Estados Unidos) mientras que en otros se realiza en la medicina pública (España, Canadá, Inglaterra). Los países con provisión privada se caracterizan por ofrecer calidades asistenciales desiguales y ser caras para el usuario, por lo que ciertos colectivos las frecuentan menos (inmigrantes de bajo poder adquisitivo, familias numerosas). En los países con provisión pública, existe buena calidad asistencial en conjunto, pero concurren graves problemas de acceso debido a la presencia de listas de espera, lo que explica que en la práctica, también muchos viajeros se marchen sin ser aconsejados ni vacunados ${ }^{7}$.

Al ser España un país de poder adquisitivo medio, las unidades privadas sólo atenderían a la población más rica. En consecuencia, conservar la provisión pública en nuestro medio tiene lógica, por lo menos en lo referente a determinación de precios de servicios y propiedad de los centros, pudiéndose entrar a discutir la privacidad de la gestión de los mismos.

\section{Elementos operativos o la táctica}

\section{Pacto con la dirección}

De manera conjunta con la dirección médica y gerencial correspondiente se debe acordar: a) los objetivos estratégicos y operacionales, b) la dotación mínima de personal, elementos informáticos, material mueble (microscopio, neveras, baterías de seguridad, etc.) y fungible, c) horario, d) sistema de programación de usuarios y, e) revisión de salario si las nuevas responsabilidades dan lugar a ello. Si la existencia de la unidad de salud internacional no es un interés compartido por asistenciales y

Tabla 1. Pros y contras de la facturación a terceros de las actividades preventivas desarrolladas en una unidad de salud internacional.

\begin{tabular}{lc}
\hline Argumentos a favor & Argumentos en contra \\
\hline - Sensibiliza a los usuarios de los costes de la asistencia. & - Da lugar a procesos de emisión de \\
- Aumenta la eficiencia de la unidad. & facturas y recibos, cobro, contabilización \\
- Permite la reinversión. & e ingreso. \\
& - Responsabilidad global del proceso \\
& indeterminada. \\
& - Da lugar a exigencias y reclamaciones de \\
& usuarios. \\
\hline
\end{tabular}


gestores, su viabilidad a medio y largo plazo es escasa.

\section{Cartera de servicios}

La correcta definición de una cartera de servicios realista, factible y sostenible es una de las claves para la viabilidad de una consulta de medicina tropical. En la tabla 2 se expone, a modo de ejemplo, la cartera de la Unitat de Salut Internacional del Barcelonès nord $i$ Maresme (USAI BniM). Como queda de manifiesto, la cartera de servicios intenta aprovechar lo que ya existe -y ésta es una idea clavemediante la consecución de alianzas-clave con servicios hospitalarios o institucionales. Además de mejorar la eficiencia, el trabajo conjunto de profesionales de diversos niveles asistenciales es algo beneficioso para el usuario y una experiencia gratificante para los propios sanitarios ${ }^{8}$.

\section{Evaluación de la calidad}

La dimensión de evaluación y control de calidad de procesos y de actividades debe estar ya presente en el diseño de la unidad. Así, es recomendable definir indi- cadores de evaluación simples y lo más objetivos posible ${ }^{9}$. En la tabla 3 se representan los indicadores y estándares de calidad definidos en la USAI BniM, con la periodicidad de su evaluación.

\section{El laboratorio}

Las pruebas microbiológicas necesarias para la atención de pacientes afectos de enfermedades importadas pueden dividirse en: a) pruebas urgentes inmediatas, b) pruebas ordinarias de alta sensibilidad para diagnóstico de casos y, c) pruebas de alta especificidad para confirmación de las anteriores o estudio de enfermedades infrecuentes.

En el primer caso, las pruebas urgentes inmediatas deben poder realizarse al momento en las propias unidades. Fundamentalmente son dos: extensión de sangre periférica y parásitos de heces en fresco para valoración de paludismo (especie y porcentaje de parasitación) y amebiasis agudas respectivamente.

Las pruebas ordinarias del segundo caso generalmente están disponible en cualquier laboratorio, inclusive la mayoría

Tabla 2. Cartera de servicios de la Unidad de Salud Internacional BniM.

a. Actividades preventivas en viajeros internacionales

Incluye la provisión de:

1. Consejos a viajeros

2. Vacunación internacional

3. Quimioprofilaxis antipalúdica (con el Servicio de Farmacia Clínica del HUGTiP)

b. Actividades preventivas del retorno

Incluye la:

1. Quimioprofilaxis secundaria de ETS, punciones accidentales y exposiciones a tuberculosis

2. Inmunoterapia antirrábica post-exposición

3. Revisiones a cooperantes o personas expatriadas durante largo tiempo o a niños adoptados procedentes de países de alta prevalencia de enfermedades endémicas tropicales (con el Servicio de Medicina Preventiva del HUGTiP)

c. Actividades asistenciales en enfermedades importadas

Visita en 24 horas de las sospechas de patología importada febril en viajeros europeos o inmigrantes (con la Unidad de Enfermedades Infecciosas del HUGTiP). Algunas pruebas complementarias consideradas urgentes inmediatas se realizan en la propia USAI BniM (ver cartera de servicios de laboratorio)

d. Unidad de tuberculosis y Salud Comunitaria

Diagnóstico, tratamiento y seguimiento de casos índice de tuberculosis en pacientes inmigrantes en situación socio-laboral inestable (con la delegación del BniM del Departament de Salut de la Generalitat de Catalunya)

HUGTiP: Hospital Universitari Germans Trias i Pujol, Badalona.

ETS: Enfermedades de transmisión sexual

BniM: Barcelonés nord i Maresme. Agrupa dos comarcas, cada una de ellas con una población de unos 350.000 habitantes. 
Tabla 3. Índices de calidad (estándar de calidad definido para el primer año).

\begin{tabular}{ll}
\hline Medida de calidad & Estándar \\
\hline Prescripción vacunal & Índice de adecuación observada (prescripción \\
& real/recomendaciones OMS). Anual $(>90 \%)$. \\
& Índice de sobrevacunación (vacuna innecesarias/ \\
totales). Anual $(<5 \%)$. & Índice de infravacunación (vacunas omitidas/ \\
& totales). Anual $(<5 \%)$ \\
& Chequeo de neveras y lotes vacunales. Mensual. \\
\hline Asistencia a enfermedades importadas & Derivaciones $(\%)$. Anual $(<25 \%)$. \\
& Ratio primeras/segundas visitas (\%). Anual. \\
\hline Atención al usuario & № y porcentaje de reclamaciones. Semestral. \\
& Días de lista de espera. Semanal $(<15$ días). \\
\hline Seguimiento de pacientes tuberculosos & Porcentaje de curaciones. Anual $(>70 \%)$. \\
& Porcentaje de cumplimiento de quimioprofilaxis \\
& secundaria. Anual $(>70 \%)$. \\
\hline
\end{tabular}

de los de atención primaria. En este sentido, se deben escoger pruebas simples técnicamente, baratas, y que puedan realizarse con el utillaje ya existente en el laboratorio. Cabe insistir en la idea de aprovechar lo que ya funciona. En la tabla 4 se ejemplifica la cartera de servicios del laboratorio (laboratorio de atención primaria) de la USAI BniM

Las pruebas de alta especificidad (algunas serologías, inmunología) implican la creación de circuitos de derivación a los hospitales de tercer nivel o universidades que las posean. Es inútil e ineficiente disponer de ellas en cada unidad.

\section{Reinformación}

La información registrada en la unidad debe ser accesible a los otros profesionales a través de la red informática existente, especialmente a la atención primaria, por lo que concierne a vacunaciones y quimioprofilaxis antipalúdica empleada. Para ello es recomendable usar el mismo programa informático que el habitual de la red sanitaria de la comunidad autónoma. Evidentemente, es necesario "personalizar" algunos aplicativos para adaptarlos funcionalmente a la realidad de la consulta de salud internacional. De esta manera, abrir desde la dirección una página específica de salud internacional es algo sencillo. Además, los informes de alta de pacientes con enfermedades importadas deben ser escritos aparte y remitidos a sus médicos de cabecera por correo electrónico o valija. Hay que recordar que la mayoría de enfermedades tropicales y las reacciones adversas a vacunas son de declaración obligatoria $^{10,11}$.

\section{Limitaciones del modelo}

$\mathrm{Al}$ modelo de unidades de salud internacional interniveles se le pueden objetar varios puntos débiles, a saber: a) aunque es un modelo más eficiente que el representado por una consulta de medicina tropical aislada, dista mucho de ser rentable en términos economicistas (coste/beneficio), b) la diversidad de administraciones (locales, autonómicas y estatales) y de proveedores, complican notablemente la consecución de determinadas vacunas y quimioprofilaxis antipalúdica, c) es dependiente tecnológicamente de la existencia de red informática sanitaria y d) existen zonas de responsabilidad "gris" debido a la no-jerarquización de los profesionales implicados en relación con sus compañeros de hospital o de atención primaria.

\section{Conclusión}

Las consultas de medicina tropical pura sólo tienen sentido en los hospitales de tercer nivel con alta tecnología. Las 
Tabla 4. Cartera de servicios en diagnóstico microbiológico USAI BniM en enfermedades importadas.

\begin{tabular}{|c|c|c|}
\hline Motivo diagnóstico & Lugar & Procedimiento \\
\hline $\begin{array}{l}\text { PALUDISMO: } \\
\text { Sospecha (urgente): } \\
\text { Protocolizado, control post-tt } \\
\text { Estudio diagnóstico } \\
\text { Estudio tratamiento } \\
\end{array}$ & $\begin{array}{c}\text { USAI BniM } \\
\text { Laboratorio CAP II } \\
\text { Laboratorio CAP II } \\
\text { Laboratorio CAP II (PC) } \\
\end{array}$ & $\begin{array}{c}\text { Gota fresca - extensión } \\
\text { Extensión } \\
\text { Serología } \\
\text { Prueba GLUC-6-P DH } \\
\end{array}$ \\
\hline $\begin{array}{l}\text { ESQUISTOSOMIASIS } \\
\text { Parásitos en orina o heces } \\
\text { Biopsia rectal }\end{array}$ & $\begin{array}{l}\text { Laboratorio CAP II } \\
\text { Obtenida en USAI }\end{array}$ & $\begin{array}{l}\text { Centrifugado orina /24h } \\
\text { valorada en Lab CAP II }\end{array}$ \\
\hline $\begin{array}{l}\text { AMEBIASIS (y otros parásitos en h } \\
\text { Amebiasis aguda } \\
\text { Cuadros subagudos } \\
\text { Cuadros crónicos }\end{array}$ & $\begin{array}{c}\text { USAI BniM } \\
\text { Laboratorio CAP II } \\
\text { Laboratorio CAP II }\end{array}$ & $\begin{array}{l}\text { Parásitos en fresco } \\
\text { Parásitos en MIF } \\
\text { Flotación ClNa } \\
\text { Auramina de heces }\end{array}$ \\
\hline ESTRONGYLOIDIASIS & Laboratorio CAP II & $\begin{array}{c}\text { Concentración de heces } \\
\text { (Baerman modificado) }\end{array}$ \\
\hline $\begin{array}{l}\text { FILARIASIS } \\
\text { Filarias hemáticas/linfáticas } \\
\text { Filarias dérmicas } \\
\end{array}$ & $\begin{array}{c}\text { Laboratorio CAP II } \\
\text { USAI BniM }\end{array}$ & $\begin{array}{c}\text { Leucoconcentración } \\
\text { Extensión en sangre } \\
\text { Prick-test }\end{array}$ \\
\hline DIARREA DEL VIAJERO & Laboratorio CAP II & Coprocultivo \\
\hline $\begin{array}{l}\text { TRIPANOSOMIASIS } \\
\text { Estudio diagnóstico } \\
\text { Estudio diagnóstico } \\
\end{array}$ & $\begin{array}{c}\text { Laboratorio CAP II } \\
\text { Laboratorio CAP II (PC) }\end{array}$ & $\begin{array}{c}\text { Punción de adenopatías } \\
\text { Serología } \\
\end{array}$ \\
\hline $\begin{array}{l}\text { DENGUE } \\
\text { Estudio diagnóstico }\end{array}$ & Laboratorio CAP II & Serología \\
\hline
\end{tabular}

USAI BniM: Unidad de Salud Internacional. Barcelonés nord i Marcéeme.

CAP II: Centro de Atención primaria II-especialidades médicas.

PC: Prueba concertada con otro laboratorio.

consultas de salud internacional responden a las necesidades preventivas y curativas derivadas de una población viajera y/o inmigrante en fuerte aumento. Su eficiencia y factibilidad depende de las relaciones con las direcciones médicas, su situación interniveles, el aprovechamiento de los recursos organizativos y asistenciales ya existentes, la implementación de programas de control de calidad y el trabajo en red informática.

\section{BIBLIOGRAFÍA}

1. Valerio L, Fabregat A, Martínez O. Unidades de salud internacional centradas en atención primaria. Cuadernos de Gestión 2001; 7: 100-105.

2. Hardling R, CRook P, Lewthwite P. Burden and cost of imported infections admitted to infectious diseases units in England and
Walles in 1998 and 1999. J Infect 2004; 48: 139-144.

3. Cetron M, Jeyston J, Shlim D, Steffen R. Traveller's Health. Emerg Infect Dis 1998; 4: 405407.

4. Fortney JC, Steffick DE, Burgués JF, MacieJEWSHI ML, PETERSEN LA. Are primary care services a substitute or a complement for specialty and inpatient services? Health Serv Res 2005; 40: 1422-1442.

5. Valerio L, Martinez O, Sabrià M, Esteve M, URBIZTONDO L, Roca C. High-risk travel abroad overtook low-risk travel from 1999 to 2004: characterization and trends in 2622 Spanish travellers. J Travel Med 2004; 12; 371-331.

6. DOGC $\mathrm{n}^{\mathrm{o}} \quad 3726-25 / 09 / 2002$. Resolució sss/2619/2002, de 2 de setembre, sobre la revisió de preus públics corresponents als serveis que presta l'Institut Català de la Salut; p. 16.696.

7. PORTER JF, KNILL-JONES RP. Quality of travel health advice in higher-education establish- 
ments in the United Kingdom and its relationship with the demographic background of the provider. J Travel Med 2004; 11: 347353.

8. Chaudet H, Pellegrin L, Boureau C, Delmont J. Evaluating he use of comprehensive pre-travel database by travel medicine experts and novices for advice building. $\mathrm{J}$ Travel Med 2004; 11; 148-154.

9. BEHRENS RH, ROBERTS JA. Is travel prophylaxis worth while? Economic appraisal of prophy- lactic measures against malaria, hepatitis A, and typhoid in travellers. BMJ 1994; 309: 918-922.

10. PACKHAM CJ. A survey of notified travel-associated infections: implications for travel health advice. J Public Health Med 1996; 18: 123-125.

11. Keystone JS, Korzasky PE, Freedman DO. Internet and computer-based resources for travel medicine practitioners. Clin Infect Dis 2001; 32: 757-765. 